\title{
Negative emotional state slows down movement speed: behavioral and neural evidence
}

\author{
Xiawen $\mathrm{Li}^{1}{ }^{1}$, Guanghui Zhang ${ }^{2}$, Chenglin Zhou ${ }^{1}$, Xiaochun Wang ${ }^{\text {Corresp. } 1}$ \\ ${ }^{1}$ Department of Psychology, Shanghai University of Sport, shanghai, China \\ 2 Faculty of Information Technology, University of Jyväskylä, Jyväskylä, Finland \\ Corresponding Author: Xiaochun Wang \\ Email address: wangxiaochun@sus.edu.cn
}

Background. Athletic performance is affected by emotional state. Athletes may underperform in competition due to poor emotion regulation. Movement speed plays an important role in many competition events. Flexible control of movement speed is critical for effective athletic performance. Although behavioral evidence showed that negative emotional can influence movement speed, the nature of the relationship remains controversial. Thus, the present study investigated how negative emotion affects movement speed and the neural mechanism underlying the interaction between emotion processing and movement control.

Methods. The present study combined electroencephalography (EEG) technology with a cued-action task to investigate the effect of negative emotion on movement speed. In total, 21 undergraduate students were recruited for this study. Participants were asked to perform six consecutive action tasks after viewing an emotional picture. Pictures were presented in two blocks (one negative and one neutral). After the participants completed a set of tasks (neutral of negative), they were subjected to complete a 9-point self-assessment manikin scale. Participants underwent EEG while performing the tasks.

Results. At the behavior level, there was a significant main effect of emotional valence on movement speed, with participants exhibiting significantly slower movements in the negative emotional condition than in the neutral condition. EEG data showed increased theta oscillation and larger P1 amplitude in response to negative than to neural images suggesting that more cognitive resources were required to process negative than neutral images. EEG data also showed a larger late CNV area in the neutral condition than in the negative condition, which suggested that there was a significant decrease in brain activation during action tasks in negative emotional condition than in the neural. While, the early CNV did not reveal a significant main effect of emotional valence.

Conclusion. The present results indicate that a negative emotion can slow movement which largely due to negative emotional processing consuming more resources than non-emotional processing and this interference effect mainly occurred in the late movement preparation phase. 
Negative emotional state slows down movement

\section{2 speed: behavioral and neural evidence}

3

4

$5 \quad{ }^{1}$ Department of Psychology, Shanghai University of Sport, Shanghai, China

6 '2Faculty of Information Technology, University of Jyväskylä, Jyväskylä,Finland

7

8 Corresponding Author:

9 Xiaochun Wang

10 Hengren Road,Shanghai,200438,China

11 Email address: wangxiaochun@sus.edu.cn

12

13

14

15

16

17

18

19

20

21

22

23

24

25

26

27

28

29

30

31

32

33

34

35

36

37 


\section{Abstract}

Background. Athletic performance is affected by emotional state. Athletes may underperform in competition due to poor emotion regulation. Movement speed plays an important role in many competition events. Flexible control of movement speed is critical for effective athletic performance. Although behavioral evidence showed that negative emotional can influence movement speed, the nature of the relationship remains controversial. Thus, the present study investigated how negative emotion affects movement speed and the neural mechanism underlying the interaction between emotion processing and movement control.

Methods. The present study combined electroencephalography (EEG) technology with a cuedaction task to investigate the effect of negative emotion on movement speed. In total, 21 undergraduate students were recruited for this study. Participants were asked to perform six consecutive action tasks after viewing an emotional picture. Pictures were presented in two blocks (one negative and one neutral). After the participants completed a set of tasks (neutral of negative), they were subjected to complete a 9-point self-assessment manikin scale. Participants underwent EEG while performing the tasks.

Results. At the behavior level, there was a significant main effect of emotional valence on movement speed, with participants exhibiting significantly slower movements in the negative emotional condition than in the neutral condition. EEG data showed increased theta oscillation and larger P1 amplitude in response to negative than to neural images suggesting that more cognitive resources were required to process negative than neutral images. EEG data also showed a larger late $\mathrm{CNV}$ area in the neutral condition than in the negative condition, which suggested that there was a significant decrease in brain activation during action tasks in negative emotional condition than in the neural. While, the early CNV did not reveal a significant main effect of emotional valence.

Conclusion. The present results indicate that a negative emotion can slow movement which largely due to negative emotional processing consuming more resources than non-emotional processing and this interference effect mainly occurred in the late movement preparation phase.

Keywords: negative emotion; movement speed; theta oscillation; P1; CNV 


\section{Introduction}

79

80

81

82

83

84

85

86

87

88

89

90

91

92

93

94

95

96

97

98

99

100

101

102

103

104

105

106

107

108

109

110

111

112

113

114

115

116

117

118

Movement speed plays an important role in sports. Flexible control of movement speed is critical for effective athletic performance. Many factors can influence movement speed, with emotional state, especially a negative emotional state, being a potentially important factor. Athletes may underperform in competition due to poor emotion regulation. Although emotion effects on movement speed have been explored previously, the nature of the relationship remains controversial. Elucidation of how negative emotion affects movement speed may be useful toward guiding athletes with respect to how to adjust modulate emotional state according to competition circumstances and regulate their movement speed accordingly.

Studies have demonstrated an intimate relationship between emotion and the motor system(Beatty et al. 2016; Simoes Matos Saraiva 2017). Specifically, emotional state has been reported to affect movement speed(Hälbig et al. 2011), including walking speed (Michalak et al. 2009), running speed(Lane et al. 2015), and even the velocity of a thrown ball (Rathschlag \& Memmert 2013). The broaden-and-build theory of positive emotions posits that positive emotion can broaden attention and cognitive range, thus promoting agility of thinking (Fredrickson 2015), while negative emotions do the opposite. In accordance with this theory, research has shown that under induced states of sadness and depression, human subjects exhibited an altered gait pattern characterized by reduced walking speeds, vertical head movements, and arm swinging (Michalak et al., 2009). In a study employing a target-detection paradigm, Pereira et al. (2006) described two types of interference effects of negative emotion on reaction speed: transient and sustained. They found that the duration of interference was related to negative emotional state stability with more stable negative states producing longer lasting interference effects (Pereira et al. 2006). It has been suggested that the impact of emotion on movement may be related to the time interval between the emotional stimulus and task-cue stimulus presentation with the effect on movement performance dissipating over time and, potentially, being fully mitigated after a sufficient time interval (Contreras et al. 2013).

From an evolutionary point of view, emotional responses may be defensive or appetitive (Bradley et al. 2001). The defensive system is activated primarily by negative stimuli triggering avoidance, while the appetitive system is activated by positive stimuli triggering approach. Hence, it is natural for people to approach a favorable stimulus and avoid a harmful one. If movement conforms to this evolutionary rule, speed might become faster (Chen \& Bargh 1999). However, unlike other negative emotions, anger has been proved to be an approach-related emotion that can activate the appetitive motivational system(Carver \& Eddie 2009). Thus, our experimental materials did not include angry emotional stimuli based on this specificity. The classic paradigm employed in examining this question requires participants to pull or push a joystick after watching a positive or a negative stimulus. Pushing and pulling motions have been found to be faster under negative and positive stimulus conditions, respectively, relative to motions made under neutral conditions (Krieglmeyer \& Deutsch 2010; Rotteveel \& Phaf 2004). Some studies have demonstrated that emotional stimuli, especially negative stimuli (disgust and fear stimuli), can activate approach or avoidance actions automatically. However, Rotteveel et al. (2004) has claimed instead that the influence of emotional stimuli on actions requires conscious 
119

120

121

122

123

124

125

126

127

128

129

130

131

132

133

134

135

136

137

138

139

140

141

142

143

144

145

146

147

148

149

150

151

152

153

154

155

156

157

158

159

160

161

162

163

164

awareness. In the present study, participants were required to view the emotional pictures before performing action tasks consciously.

Other prevailing ideas claim that the effect of emotion on movement depends on the specific category of emotion(Campo et al. 2016; Rathschlag \& Memmert 2015). For example, participants threw a ball faster when instructed to recall feelings of anger or happiness than in an emotionally neutral state. Meanwhile, participants jumped higher under anger and happiness conditions than under anxiety and sadness conditions (Rathschlag \& Memmert 2013). Taken together, positive emotion has been shown to benefit movement speed control consistently, while the influence of negative emotion on movement speed is more controversial on behavioral level.

It has been shown that some brain circuits responsible for movement control are also involved in emotional processing(Stoodley \& Schmahmann 2010). Brain circuits which was involved in emotional processing are activated during the movement of athletics (Lang et al. 1998). Viewing emotional pictures has been reported to alter motor cortex excitability (Coombes et al. 2009), and negative emotion has been suggested to facilitate primary motor cortex plasticity by modulating intracortical GABAergic neurotransmission (Koganemaru et al. 2012). However, electroencephalography (EEG) evidence related to these effects is quite limited. More evidence is needed to understand the neural mechanism underlying the interaction between emotion and movement control.

In the current study, we combined EEG technology with a cued-action task to investigate the effect of negative emotion on movement speed, the duration of this effect, and the ERP correlates of such an interaction. The task used in this study was a combination of the targetdetection paradigm developed to investigate how viewing task-irrelevant emotional pictures affects the performance of a detection task (Pereira et al. 2006) and a cued-target paradigm, the classic contingent negative variation (CNV) induced paradigm(Rockstroh et al. 1982; Walter 1964). Unlike the simple button press task of studying response times, the present action task was more complex including three consecutive finger actions and thus more suitable to examine the movement speed. The study analyzed theta oscillation and P1 component in the emotional image processing period and the CNV component in the action task period. CNV has been related to movement preparation for such that $\mathrm{CNV}$ amplitude is enhanced when more attention is allocated or an movement is well prepared (Carretié et al. 2007; Walter 1964). CNV potential consists of early and late CNV arising from the frontal and parietal regions, respectively(Rohrbaugh et al. 1976). The early CNV potential indexes cognitive processing such as sensory preparation, anticipatory attention to the forthcoming target stimuli (Gómez et al. 2004; Loveless \& Sanford 1974). The late CNV potential corresponds to movement preparation(Anatürk \& Jentzsch 2015; Leuthold et al. 2006). In addition, occipital theta oscillation and P1 was reported on the presentation of emotion images and researchers observed increased theta and P1 response to negative stimuli(Aftanas et al. 1998; Flaisch et al. 2010; Güntekin \& Başar 2014; Meng et al. 2016). We hypothesized that negative emotion would have an interference effect on movement speed. Specifically, we predicted that our negative emotion condition would be associated with a slowing of movement speed, theta oscillation and P1 increasing and $\mathrm{CNV}$ weakening relative to the neutral condition.

\section{Materials \& Methods Participants}

A total of 21 undergraduate volunteers (10 males, 11 females; $21 \pm 2.21$ years) from Shanghai University of Sports participated in this experiment. They were all right-handed, with 
165

166

167

168

169

170

171

172

173

174

175

176

177

178

179

180

181

182

183

184

185

186

187

188

189

190

191

192

193

194

195

196

197

198

199

200

201

202

203

204

205

normal or corrected-to-normal vision, and had no self-reported history of mental illness or chronic physical illness. All of them were paid a modest compensation after the experiment. This experiment was approved by the ethics committee of the Shanghai University of Sport (No. 2015007) and written informed consent was obtained from all participants.

\section{Materials}

We selected 310 emotional images from the Chinese Affective Picture System (Lu et al. 2005) and International Affective Picture System (Lang 1999), half of which were negative and half neutral. The formal experiment utilized 150 negative and 150 neutral images, and the remaining 10 images were used for practice. Images with the same emotional valence were presented together to induce a stable emotional state (Larson et al. 2005). Each picture had been assessed for its valence and arousal on a 9-point self-assessment manikin (SAM) scale. The paired t-test performed on the average SAM scores showed that there was a significantly difference between negative images and neutral images in emotional valence $(p<0.001)$ and arousal $(p<0.001)$ (Table1).Negative images had an average pleasure rating of 2.63 and an average arousal rating of 5.88; the neutral images ratings were 5.03 and 3.70, respectively.

\section{Design and procedure}

The experiment employed a $2 \times 2$ within-subject design with emotional valence (negative vs. neutral) and action task sequence (1st and 6th action) as the factors. In the cued-action task, participants were prompted to perform six consecutive action tasks after viewing an emotional picture. Each emotional valence condition consisted of 150 pictures. The task data were compiled by E-Prime 2.0 software.

After being introduced to the cued-action task, participants pressed and held key "2". Simultaneously, an emotional image was presented for 2000-ms. Participants were instructed to watch this image carefully. The image was followed by an $800-\mathrm{ms}$ presentation of a fixation point. Then, a circle appeared around the fixation point to indicate to the participant that an action was required; once the circle appeared (after 800-ms fixation point), participants were expected to release key "2" immediately, press key " 5 ", and then return to holding key " 2 ". After participants returned to holding key " 2 ", a black screen was presented for 500-ms. This sequence was the first action task. The 800-ms fixation point-action task cue (i.e. circle) 500 -ms black screen cycle was repeated five more times resulting in six total action tasks (Fig.1). This number of action tasks was selected based on previously published work (Pereira et al. 2006), wherein it was shown that the analysis of time-separated actions in a series can be used to distinguish sustained from transient interference effects of negative emotion on reaction speed. Only when participants completed the last action task (sixth action task) successfully was the next picture presented. The order of negative and neutral blocks was counterbalanced across participants. After the participants completed a set of tasks (neutral of negative), they were subjected to a 9point self-assessment manikin scales. The entire experiment was performed in a dimly lit, sound attenuated room. 
206

207

208

209

210

211

212

213

214

215

216

217

218

219

220

221

222

223

224

225

226

227

228

229

230

231

232

233

234

235

236

237

238

239

240

241

242

243

244

245

246

247

248

249

\section{Behavior data analyses}

Behavioral data of action time were recorded by E-Prime 2.0, including the time spent on the first and the sixth action tasks (the total time it took to release key 2, press 5, release 5, and press 2 again). Data falling beyond three standard deviations of the mean were excluded. Statistical analysis of action time was performed in SPSS 22.0. Data were analyzed by two-way repeated measures analyses of variance (rmANOVAs) with emotional valence (negative and neutral) and action task sequence (1st and 6th action). In order to improve the estimates of the effect, we also conducted a two-way rmANOVAs with emotional valence (negative and neutral) and action task sequence (1st, 2nd, 3rd ,4th ,5th and 6th action).

The scores of the 9-point emotional self-assessment were analyzed by the paired t-test with emotional valence (negative and neutral).

\section{EEG data acquisition and analysis}

EEG measures electrical brain responses directly and with high temporal resolution. EEG was conducted with $64 \mathrm{Ag}-\mathrm{AgCl}$ electrodes arranged according to the international 10-20 system with a sampling frequency of $1000 \mathrm{~Hz}$ (Brain Products $\mathrm{GmbH}$, Germany). The EEG was recorded referentially against the $\mathrm{FCz}$, and $\mathrm{AFz}$ served as the ground electrode. The vertical electrooculogram was recorded infra-orbitally at the left eye and the horizontal electrooculogram was recorded latera-orbitally of the right eye. All electrooculogram and electroencephalogram electrodes impedances were maintained below $5 \mathrm{k} \Omega$.

EEG data analysis includes two parts: emotional image processing analysis and action preparation analysis. The EEG were analyzed off-line by EEGLAB in the MATLAB environment. FCz was re-referenced to the average of TP9 and TP10. Then, ocular artifacts were removed through independent component analysis. Next, we remove line noise with a $50 \mathrm{hz}$ notch filter. Then, the data were filtered with a $30-\mathrm{Hz}$ low-pass cutoff and a $0.5 \mathrm{~Hz}$ high-pass cutoff, respectively.

Time-domain and time-frequency analysis were used to analyze the emotional image processing data. The data were extracted offline from 200 -ms pre-image onset to 2000 -ms postimage onset. All epochs were baseline-corrected with respect to the mean voltage over the $200 \mathrm{~ms}$ preceding image onset, epochs with signals that exceeded $\pm 100 \mu \mathrm{V}$ were rejected $(95.68 \%$ of the trials was retained on average per participant), then averaged by experimental condition. For time-domain analysis, Fast Fourier transform and temporal-Principal Component Analysis (FFT and t-PCA) were performed on data processing (Achim \& Marcantoni 1997; Dien 2010; Dien 2012). The data were first filtered with a $0.5 \sim 30 \mathrm{~Hz}$ bandpass, and then detection and quantification of the desired ERP components were achieved through a matrix based on t-PCA. $\mathrm{P} 1$ at PO3, PO4, PO7,PO8,POz,O1,O2 and Oz within the time window of 190-230-ms was selected as target ERP components(Luo et al. 2010; Müller \& Gundlach 2017). Averaged P1 amplitude of these electrode was analyzed by the paired t-test with emotional valence (negative and neutral). We also selected frontal electrode F3, F4, F5, F6 and Fz to investigated activation differences in different brain regions and conducted a two-way rmANOVAs with emotion valence (negative and neutral) and brain region (frontal and occipital-temporal).

For time-frequency analysis, a complex Morlet continuous wavelet transform (CMCWT) based on the complex wavelet transform (Tallon-Baudry et al. 1996) was used for timefrequency analysis of the average ERP data in the MATLAB. CMCWT was described as 
250

251

252

253

254

255

256

257

258

259

260

261

262

263

264

265

266

267

268

269

270

271

272

273

274

275

276

277

278

279

280

281

282

283

284

285

286

287

288

$C M C W T(t, f)=\left|(f)^{*} x(t)\right|^{2}$. The time-frequency energy CMCWT $(t, f)$ was used to calculate the convolution of the mother wavelet $\Phi(\mathrm{t}, f c)$ with the ERP data $x(t)$. Here, $\Phi(\mathrm{t}, f c)$ is the complex Morlet wavelet defined as $\Phi(\mathrm{t}, f c)=\frac{1}{\sqrt{\pi \sigma^{2}}} e^{i 2 \pi t f c} e^{\frac{-t^{2}}{2 \sigma^{2}}}(f c$, center frequency; $\sigma$, bandwidth). A wavelet family was characterized by the constant ratio $K=f c / \sigma_{f}=2 \pi \sigma f c$ with $\mathrm{K}$ being greater than5(Zhang et al. 2017). A baseline correction using the 200-ms preceding image onset again was then conducted. The PO3, $\mathrm{PO} 4, \mathrm{PO} 7, \mathrm{PO} 8, \mathrm{POz}, \mathrm{O} 1, \mathrm{O} 2$ and $\mathrm{Oz}$ electrode were selected for the analysis of the evoked theta oscillation $(5-9 \mathrm{~Hz})$ within the time window of $100-200-\mathrm{ms}$ based on previous study(Aftanas et al. 2002). Theta oscillation was analyzed by the paired t-test with emotional valence (negative and neutral). We also observed frontal activation at F3, F4, F5, F6 and Fz as well as P1. Two-way rmANOVAs was used to analyze averaged theta oscillations power with emotion valence (negative and neutral) and brain region (frontal and occipitaltemporal).

For action preparation analysis, the data were segmented from 800-ms prior to the onset of the action task cue (circle) to 1000-ms after stimulus onset. All epochs were baseline-corrected with respect to the mean voltage over the $-800 \sim-700$-ms period preceding stimulus onset, then averaged by experimental condition. CNVs were selected as target ERP components. We analyzed the average early CNV area within the time window of -200-ms $\sim 0-\mathrm{ms}$ at Fz and at the late $\mathrm{CNV}$ area within the time window of $0 \sim 140-\mathrm{ms}$ at FCz (Rohrbaugh et al. 1976). After data preprocessing, data of one participant was removed due to many additional movements. We then averaged the participants' data for each period. The average of early CNV and the averaged late CNV data were analyzed by two-way rmANOVAs with emotional valence (negative and neutral) and action task sequence (1st and 6th action) based on the behavioral results.

\section{Results}

\section{Behaviors}

Emotional self-assessment data results showed that the SAM scores in negative condition were significantly less than neutral condition $(p<0.001)$ (Fig.2A). We used scatter plots with box plots to improve data transparency in Fig.2B.

Behavioral data results of $2 \times 2$ rmANOVAs showed a main effect of both action sequence $\left(\mathrm{F}_{1,19}=49.807 ; p<0.001 ; \eta_{P}^{2}=0.724\right)$ and emotional valence $\left(\mathrm{F}_{1,19}=6.826 ; p=0.017 ; \eta_{P}^{2}=\right.$ 0.264 ) on action time. The duration of the sixth action was significantly shorter than that of the first, and action time in the negative condition was significantly longer than that in the neutral condition. However, there was not a significant interaction of these two factors $\left(\mathrm{F}_{1,19}=0.329 ; p\right.$ $=0.573 ; \eta_{P}^{2}=0.017$ ) (Fig.2C). The scatter plots with box plots were showed in Fig.2D.

Behavioral data results of $2 \times 6 \mathrm{rmANOVAs}$ showed a main effect of both action sequence $\left(\mathrm{F}_{5,15}=17.467 ; p<0.001 ; \eta_{P}^{2}=0.853\right)$ and emotional valence $\left(\mathrm{F}_{1,19}=7.205 ; p=0.015 ; \eta_{P}^{2}=\right.$ $0.275)$ on action time. there was not a significant interaction of these two factors $\left(\mathrm{F}_{5,15}=2.804 ; p\right.$ $\left.=0.055 ; \eta_{P}^{2}=0.483\right)$. The pairwise comparison found that the first action time was significantly slower than next 5 action task (2nd, 3rd ,4th ,5th,6th $)(p<0.01)$; while there was no significant 
289

290

291

292

293

294

295

296

297

298

299

300

301

302

303

304

305

306

307

308

309

310

311

312

313

314

315

316

317

318

319

320

321

322

323

324

325

difference between the sixth action task and the previous four action tasks $(2 \mathrm{nd}, 3 \mathrm{rd}, 4 \mathrm{th}, 5 \mathrm{th}$, (Fig.3).

\section{EEG \\ P1 evoked by emotional image}

P1 brain topography illustrated that this effect was particularly robust in the occipitaltemporal cortex. Paired t-test result showed that negative stimuli evoking larger P1 amplitude than neutral stimuli in the occipital-temporal $(p=0.002)($ Fig. $4 A) .2 \times 2$ rmANOVAs of emotion and brain region showed a main effect of brain region $\left(\mathrm{F}_{1,19}=135.202 ; p<0.001 ; \eta_{P}^{2}=0.877\right)$, but not emotion $\left(\mathrm{F}_{1,19}=3.449 ; p=0.079 ; \eta_{P}^{2}=0.154\right)$. $\mathrm{P} 1$ evoked in occipital-temporal was significantly larger than that evoked in frontal region. There was a significant interaction between these two factors $\left(\mathrm{F}_{1,19}=8.446 ; p=0.009 ; \eta_{P}^{2}=0.308\right)$ (Fig. 4 B and Fig. 4C).

\section{Theta oscillation evoked by emotional image}

Brain topography of the evoked theta oscillation $(5-9 \mathrm{~Hz})$ was shown in Fig.5C and Fig.5D. Overt theta oscillations were present at $100-200-\mathrm{ms}$ in negative and neutral condition in occipital-temporal region. Paired t-test result showed that negative stimuli evoking larger theta oscillation than that of neutral stimuli $(p=0.006)($ Fig. $5 A$ and Fig. $5 B) .2 \times 2$ rmANOVAs of emotion and brain region revealed a main effect of brain region $\left(\mathrm{F}_{1,19}=9.402 ; p=0.006 ; \eta_{\mathrm{P}}{ }^{2}=\right.$

$0.331)$ and emotion $\left(F_{1,19}=10.875 ; p=0.004 ; \eta_{P}^{2}=0.364\right)$. Theta oscillation evoked in occipitaltemporal was significantly larger than that evoked in frontal region (Fig.5C and Fig.5D). Negative stimuli evoked larger theta oscillation than that of neutral stimuli. There was not a significant interaction between these two factors $\left(\mathrm{F}_{1,19}=1.725 ; p=0.205 ; \eta_{P}^{2}=0.083\right)$.

\section{Early CNV}

ERP results revealed a main effect of action sequence $\left(\mathrm{F}_{1,19}=7.084 ; p=0.015 ; \eta_{P}^{2}=0.272\right)$, but not emotion $\left(\mathrm{F}_{1,19}=2.277 ; p=0.148 ; \eta_{P}^{2}=0.107\right)$, on early $\mathrm{CNV}$, with the sixth action condition evoking a larger early $\mathrm{CNV}$ area than the first action(Fig. $6 \mathrm{~A})$. There was not a significant interaction between these two factors $\left(\mathrm{F}_{1,19}=0.874 ; p=0.361 ; \eta_{P}^{2}=0.044\right)$. Early $\mathrm{CNV}$ brain topography illustrated that this effect was particularly robust in the frontoparietal region (Fig.6C, Fig. 6D, Fig.6E, and Fig. 6F).

\section{Late CNV}

ERP results revealed a main effect of both emotion $\left(\mathrm{F}_{1,19}=5.644 ; p=0.028 ; \eta_{P}^{2}=0.229\right)$ and action sequence $\left(\mathrm{F}_{1,19}=22.891 ; p<0.001 ; \eta_{P}^{2}=0.546\right)$, on the late $\mathrm{CNV}$, with a larger $\mathrm{CNV}$ area in neutral condition than in negative condition and a larger CNV area in sixth action condition than in first action condition (Fig. 6B). There was not a significant interaction between these two factors $\left(\mathrm{F}_{1,19}=0.347 ; p=0.564 ; \eta_{P}^{2}=0.018\right)$. Late CNV brain topography illustrated 
326

327

328

329

330

331

332

333

334

335

336

337

338

339

340

341

342

343

344

345

346

347

348

349

350

351

352

353

354

355

356

357

358

359

360

361

362

363

364

365

that this effect was also particularly robust in the frontoparietal region (Fig.6G, Fig.6H, Fig.6I, and Fig. 6J).

\section{Discussion}

The present results support our hypothesis that negative emotion can have an interference effect on movement speed. Our study demonstrates that viewing negative emotion images can induce negative emotion which significantly slowed movement speed compared with that under neutral condition. These behavioral effects were accompanied by significant changes in ERP component. Negative images evoked larger P1 and theta oscillation than neutral images during emotion processing period. Late CNV differed significantly between emotion conditions and action sequence conditions in the action task period. Interestingly, early CNV which is related to cognitive processing was only differed significantly between action sequences rather than emotion conditions.

On the behavioral level, it was shown that viewing negative emotion images clearly induce negative emotion, consistent with the prior finding showing that the magnitude of negative emotion produced by negative images increases as participants view more emotionally negative images (Pereira et al. 2006). As a consequence, movement speed in the negative emotion was slowed, compared with that in neutral condition. Our findings are consistent with previous researches claiming that negative emotion may interfere with behavioral performance (Gross et al. 2012; Pv et al. 2014). In the negative emotional condition, movement speed was slowed down in the both first action and sixth action task. This means the duration of this slow down effect can be maintained until the end of six action tasks. This interference effect is probably caused by the induced negative emotion. Fredrickson (2015) has shown that individuals in a negative emotional state have a narrower range of cognitive resources, which in turn has a negative impact on individual behavior. Pereira et al. (2006) reported both transient and sustained interference effects of negative emotion on a target-detection task. The interference on movement speed can be explained as a reduced availability of cognitive resources (Fredrickson 2015; Melcher et al. 2012; Van et al. 2011), thus disturbing the action task. People are very sensitive to negative stimuli (Lu et al. 2015; Yuan et al. 2007), with negative stimuli being more likely to attract people's attention and thus more likely to be encoded well (Zhang et al. 2014). Attentional blink is a phenomenon wherein people tend to devote more attention to the first stimulus than the second when two stimuli are presented in series. However, Anderson et al. (2001) observed that if the second stimulus was negative, people were less likely to forget it (Anderson \& Phelps 2001), consistent with the notion that negative stimuli tend to attract attention.

In addition to the explanation that negative emotion reduced the availability of cognitive resources, the interference effect on the first action task can be explained from a biological evolutionary perspective. Human behavior is motivated by defensive and appetitive systems (M. M. Bradley, Codispoti, Cuthbert, \& Lang, 2001); the defensive system is activated primarily by negative stimuli triggering avoidance, while the appetitive system is activated by positive stimuli 
366 triggering approach. It is natural for people to approach a favorable stimulus and avoid a harmful

367

368

369

370

371

372

373

374

375

376

377

378

379

380

381

382

383

384

385

386

387

388

389

390

391

392

393

394

395

396

397

398

399

400

401

402

403

404

405

stimulus. Once people are instructed to approach a negative stimulus, the movement speed will slow down (Chen \& Bargh 1999; Rotteveel \& Phaf 2004). Our action task can be viewed as an approach movement to the images (Maxwell \& Davidson 2010). 2004). If participants are going to approach a negative image, the action would be more disturbed than a neutral image.

Behavioral results also found that movement speed was significantly slower in the first action task than in the sixth action task. This effect may be partly due to images presented before the first action (interference of the first action) and partly due to the practice effect (promotion of the six action). Participants were easily distracted by task-irrelevant stimuli (images), which interferes with the processing of the upcoming action-cued target (Contreras et al. 2013), and thus affects movement speed. Thus, less attention resources were devoted to the first action task than the sixth action task. Posner et al. (2012) argued that in order to orient to a new task, individuals first had to disengage from what they were currently focusing on (Posner \& Petersen 2012). The time interval between the emotion stimuli and action-cued stimuli( $800-\mathrm{ms})$ in our experiment was within the purported attentional range period (500-800-ms) (Müller et al. 1998). The presumption is that such interference effects are due to participants' attention being partially consumed by the emotional images preceding the first action task. Our present observation of an interference effect in a relatively long time interval of 800 -ms suggests that the attentional range may persist for a period of at least $800-\mathrm{ms}$. In addition, the repetition of action tasks will lead to a practice effect which may also improve the movement speed. Thus, both the interference effect of images before the first action task and the practice effect on the sixth action task will make participants to perform the sixth action task faster than perform the first action task. ERP data will further reveal the brain processing mechanisms underlying this behavior.

The behavioral data of $2 \times 6 \mathrm{rmANOVAs}$ showed a more comprehensive picture of how negative emotion affected movement speed. In all six action tasks, movement speed in negative conditions was significantly slower than that in neutral conditions which is in consistent with that in $2 \times 2$ rmANOVAs. According to the comparison between different action sequences (there was no significant difference between the last four action time), we speculated that the sixth action had reached a steady state and the brain processing mechanism was similar between the last four action tasks. That's why we chose $2 \times 2$ rmANOVAs in the movement related ERP analysis

At the neural level, we observed distinct time-domain P1 component in the time window of 190-230-ms and theta oscillations $(5-9 \mathrm{~Hz})$ in the time window of 100-200-ms during the emotion processing period. The present brain topography for $\mathrm{P} 1$ and theta oscillations results suggest that the activating region was located in the occipital-temporal cortex, which is responsible for visual processing (Wong et al. 2009).The results showed that the P1 and theta oscillations elicited in the negative condition was significantly larger than that in the neutral condition. P1 was a occipitaltemporal positive component elicited by the onset of emotional faces and scenes images(Luo et al. 2010; Müller \& Gundlach 2017). Relative to neutral images, negative images elicited a larger positive P1 amplitude(Hammerschmidt et al. 2018; Luo et al. 2010). Flaisch et al.(2010) also

Peer) reviewing PDF | (2019:04:37159:2:0:NEW 27 Jul 2019) 
406 found that negative gestures elicited increased P1 than neutral gestures (Flaisch et al.

407 2010).Researchers also found that $P 1$ was regulated by attention resources (Taylor 2002). Thus,

408 the present larger P1 component elicited by negative stimuli suggesting that negative image

409 processing consume more attention resources.

410 Past studies have shown that evoked theta oscillations in the occipital-temporal cortex was

411 sensitive to emotional valence and arousal (Aftanas et al. 2002). Elevated parietal-occipital theta

412 responses were found on presentation of negative stimuli (Balconi et al. 2009). Sun et al. (2012)

413 also found that negative stimuli elicit higher theta responses than neutral cues(Sun et al. 2012).

414 However, researchers also reported increased theta response on cognitive load in frontal

415 area(Güntekin \& Başar 2009; González-Roldan et al. 2011) Hence, considered in the context of

416 the present study, a larger P1 and theta oscillations elicited in the negative condition suggest that

417 more cognitive resources are allocated to negative image processing than to neutral image

418 processing, reducing the availability of attention resources for the action tasks, leading to a

419 reduction of movement speed.

420 Consistent with the behavioral finding that movement speed was significantly faster in the

421 sixth action task than in the first, ERP data revealed a larger CNV component (early CNV and

422 late $\mathrm{CNV}$ ), in the sixth action task than that in the first. Past studies have shown that CNV was

423 related to anticipatory, attention distribution and movement preparation (Carretié et al. 2007;

424 Walter 1964). The CNV results suggested that participants paid more attention to the sixth action

425 task than that in the first action task. In the present experiment, images were presented before the

426 first action cue, while the sixth action was preceded by a blank screen. The presence of images

427 was a disturbance to the first action task. Participants might not be able to disengage their

428 attention from images to the first action cue. Previous studies have shown a response

429 slowing(Anatürk \& Jentzsch 2015) and an increase in CNV(Arjona et al. 2014) in trials preceded

430 by valid cues compared to invalid cues, consistent with our results. Huiyan et al. (2014) provides

431 evidence that uncertain cues produced smaller early contingent negative variation (CNV) than

432 did the certain cues about upcoming task. Their CNV results are also in accordance with their

433 behavioral findings that participants performed better under certain cues than uncertain

434 cues(Huiyan et al. 2014). Furthermore, the preparation phase for the sixth action started from -

435 600-ms before the target onset, 400-ms earlier than the first action (-200-ms) (Figure3). This

436 finding proved that the sixth action was well prepared than the first action.

437 Brain topography for CNVs showed that CNV activating region was centered over the

438 parieto-frontal cortex, an area that is important for attentional processes as well as motor

439 planning, preparation, and execution. The CNV component has been shown to be closely linked

440 with attention(Faugeras \& Naccache ; Tecce 1972), motor preparation (Carretié et al. 2007).

441 Participants were in the motor preparation phase throughout the time period preceding

442 presentation of the task cue (circle). The time window of the CNV corresponded exactly to this

443 preparation period. Previous studies have shown that the larger the amplitude elicited during

444 movement preparation, the faster the resulting action was(Anatürk \& Jentzsch 2015; Walter 
445 1964), consistent with our results. Therefore, our significant CNV component findings may

446

447

448

449

450

451

452

453

454

455

456

457

458

459

460

461

462

463

464

465

466

467

468

469

470

471

472

473

474

475

476

477

478

479

480

481

482

483

484

reflect the participation of $\mathrm{CNV}$ in movement preparation and attention distribution.

Interestingly, the main effect of emotion condition was only observed in the late CNV component, with neutral condition elicited larger late CNV amplitude than that elicited in the negative condition. Past studies have shown that late CNV evoked in the parietal cortex was closely related to movement preparation(Gaillard 1985). Enough attention resources must be involved in order to complete the movement with high quality (Shaw et al. 2018). CNV has been proven to be positively related to the attention allocation. Study have shown that individuals with attention deficit evoked smaller CNV amplitude than that evoked by normal people in attention related task (Albrecht et al. 2014). Researchers also found that attention training, like mindfulness, could increase the activation of CNV(Bostanov et al. 2018). Thus, CNV is always considered as a measure of increased concentration. However, evidence showed that attention resource allocation to negative stimuli was significantly different with neutral contents(Buodo et al. 2002). Vanlessen et al. (2015) using an anti-saccade task revealed that neutral condition evoked larger CNV component than affective condition(Vanlessen et al. 2015). Cudo et al. (2018) also demonstrated that CNV amplitude was less negative in the high than in the lowapproach motivated affect(Cudo et al. 2018). Hence, the present late CNV effect suggests that more cognitive resources were required when processing negative emotion, disturbing the motor preparation phase of the simultaneous action task.

The brain topography results of late CNV suggest that the activating region was located in the parietal cortex, which is responsible for movement processing (Wong et al. 2009).Considered in the context of the present study, a smaller late CNV elicited in the negative condition suggests that more cognitive resources are allocated to negative emotion processing, than to neutral processing, reducing the availability of attention resources for the action tasks, leading to a reduction of movement speed.

The present study had a few limitations. Firstly, the total number of participants was relatively small. The results might be influenced by individual differences. Secondly, in order to ensure an induced negative emotion, participants were subject to viewing 150 images. Each image was followed by six action task, which may lead to a fatigue effect. Thirdly, participants were subjected to press key 2 all the time while viewing images as mentioned above. This press action particularly disturbed the EEG signal extraction. In addition, the present study did not observe interaction of emotional valence and action sequence. We can increase the number of action tasks (like 12 action tasks) after each emotional image in the future research.

\section{Conclusions}

Our study demonstrates that movement speed was slowed in the negative emotional state. In addition, our ERP results support that the cognitive resources consumed by negative emotion processing may diminish the availability of attentional resources for action tasks. Taken together, the present behavioral and EEG data in the present study suggest that negative emotion has an interference effect on movement speed.

Peer) reviewing PDF | (2019:04:37159:2:0:NEW 27 Jul 2019) 


\section{References}

490

491

492

493

494

495

496

497

498

499

500

501

502

503

504

505

506

507

508

509

510

511

512

513

514

515

516

517

518

519

520

521

522

523

524

525

526

527

528

529

530

531

532

533

Achim A, and Marcantoni W. 1997. Principal component analysis of event-related potentials: misallocation of variance revisited. Psychophysiology 34:597-606.

Aftanas LI, Lotova NV, Koshkarov VI, and Popov SA. 1998. Non-linear dynamical coupling between different brain areas during evoked emotions: An EEG investigation. Biological Psychology 48:121-138.

Aftanas LI, Varlamov AA, Pavlov SV, Makhnev VP, and Reva NV. 2002. Time-dependent cortical asymmetries induced by emotional arousal: EEG analysis of event-related synchronization and desynchronization in individually defined frequency bands. International Journal of Psychophysiology 44:67-82.

Albrecht, Daniel B, Henrik UVS, Lilian V, Hartmut H, Xiaohui X, Renate D, Alexander H, Jonna K, and Müller UC. 2014. Genetics of preparation and response control in ADHD: the role of DRD4 and DAT1. Journal of Child Psychology \& Psychiatry 55:914-923.

Anatürk M, and Jentzsch I. 2015. The effects of musical training on movement pre-programming and re-programming abilities: An event-related potential investigation. Biological Psychology 106:39-49.

Anderson AK, and Phelps EA. 2001. Lesions of the human amygdala impair enhanced perception of emotionally salient events. Nature 411:305-309.

Arjona A, Escudero M, and Gómez CM. 2014. Updating of Attentional and Premotor Allocation Resources as function of previous trial outcome. Scientific Reports 4:4526.

Balconi M, Brambilla E, and Falbo L. 2009. BIS/BAS, cortical oscillations and coherence in response to emotional cues. Brain research bulletin 80:151-157.

Beatty GF, Cranley NM, Carnaby G, and Janelle CM. 2016. Emotions Predictably Modify Response Times in the Initiation of Human Motor Actions: A Meta-Analytic Review. Emotion 16:237.

Bostanov V, Ohlrogge L, Britz R, Hautzinger M, and Kotchoubey B. 2018. Measuring Mindfulness: A Psychophysiological Approach. Frontiers in Human Neuroscience 12:249-.

Bradley MM, Codispoti M, Cuthbert BN, and Lang PJ. 2001. Emotion and motivation I: defensive and appetitive reactions in picture processing. Emotion 1:276-298.

Buodo G, Sarlo M, and Palomba D. 2002. Attentional Resources Measured by Reaction Times Highlight Differences Within Pleasant and Unpleasant, High Arousing Stimuli. Motivation \& Emotion 26:123-138.

Campo M, Champely S, Lane AM, Rosnet E, Ferrand C, and Louvet B. 2016. Emotions and performance in rugby. Journal of Sport \& Health Science:S2095254616300369.

Carretié L, Hinojosa JA, Lópezmartín S, and Tapia M. 2007. An electrophysiological study on the interaction between emotional content and spatial frequency of visual stimuli. Neuropsychologia 45:1187-1195.

Carver CS, and Eddie HJ. 2009. Anger is an approach-related affect: evidence and implications. Psychological Bulletin 135:183-204.

Chen M, and Bargh JA. 1999. Consequences of automatic evaluation: Immediate behavior predispositions to approach or avoid the stimulus. Personality \& Social Psychology Bulletin 25:215--224.

Contreras D, Megías A, Maldonado A, Cándido A, and Catena A. 2013. Facilitation and interference of behavioral responses by task-irrelevant affect-laden stimuli. Motivation \&

Peer] reviewing PDF | (2019:04:37159:2:0:NEW 27 Jul 2019) 
534

535

536

537

538

539

540

541

542

543

544

545

546

547

548

549

550

551

552

553

554

555

556

557

558

559

560

561

562

563

564

565

566

567

568

569

570

571

572

573

574

575

576

577

578

579

580

581

582

583

584

Emotion 37:496-507.

Coombes SA, Tandonnet C, Fujiyama H, Janelle CM, Cauraugh JH, and Summers JJ. 2009. Emotion and motor preparation: A transcranial magnetic stimulation study of corticospinal motor tract excitability. Cognitive Affective \& Behavioral Neuroscience 9:380-388.

Cudo A, Francuz P, Augustynowicz P, and Stróżak P. 2018. The Effects of Arousal and Approach Motivated Positive Affect on Cognitive Control. An ERP Study. Frontiers in Human Neuroscience.

Delorme A, and Makeig S. 2004. EEGLAB: an open source toolbox for analysis of single-trial EEG dynamics including independent component analysis. Journal of neuroscience methods 134:9-21.

Dien J. 2010. Evaluating two-step PCA of ERP data with Geomin, Infomax, Oblimin, Promax, and Varimax rotations. Psychophysiology 47:170-183.

Dien J. 2012. Applying principal components analysis to event-related potentials: a tutorial. Developmental Neuropsychology 37:497.

Faugeras F, and Naccache L. Dissociating temporal attention from spatial attention and motor response preparation: A high-density EEG study. Neurolmage.

Flaisch T, Häcker F, Renner B, and Schupp HT. 2010. Emotion and the processing of symbolic gestures: an event-related brain potential study. Social cognitive and affective neuroscience 6:109-118.

Fredrickson BL. 2015. The role of positive emotions in positive psychology: The broaden-andbuild theory of positive emotions. American Psychologist 56:1367-1378.

Gómez CM, Fernández A, Maestú F, Amo C, González-Rosa JJ, Vaquero E, and Ortiz T. 2004. Task-specific sensory and motor preparatory activation revealed by contingent magnetic variation. Cognitive Brain Research 21:59-68.

Güntekin B, and Başar E. 2009. Facial affect manifested by multiple oscillations. International Journal of Psychophysiology 71:31-36.

Güntekin B, and Başar E. 2014. A review of brain oscillations in perception of faces and emotional pictures. Neuropsychologia 58:33-51.

Gaillard AK. 1985. Slow brain potentials preceding task performance. Biological Psychology 21:282-283.

González-Roldan AM, Martínez-Jauand M, Muñoz-García MA, Sitges C, Cifre I, and Montoya P. 2011. Temporal dissociation in the brain processing of pain and anger faces with different intensities of emotional expression. PAIN® 152:853-859.

Gross MM, Crane EA, and Fredrickson BL. 2012. Effort-Shape and kinematic assessment of bodily expression of emotion during gait. Human Movement Science 31:202-221.

Hälbig TD, Borod JC, Frisina PG, Tse W, Voustianiouk A, Olanow CW, and Gracies JM. 2011. Emotional processing affects movement speed. Journal of Neural Transmission 118:1319-1322.

Hammerschmidt W, Kulke L, Broering C, and Schacht A. 2018. Money or smiles: Independent ERP effects of associated monetary reward and happy faces. PloS one 13:e0206142.

Huiyan L, Hongwei G, Jin Y, Jiafeng L, Junpeng M, Nan Y, Huan X, and Hua J. 2014. Larger N2 and smaller early contingent negative variation during the processing of uncertainty about future emotional events. International Journal of Psychophysiology 94:292-297.

Koganemaru S, Domen K, Fukuyama H, and Mima T. 2012. Negative emotion can enhance human motor cortical plasticity. European Journal of Neuroscience 35:1637-1645.

Krieglmeyer R, and Deutsch R. 2010. Comparing measures of approach-avoidance behaviour: The manikin task vs. two versions of the joystick task. Cognition \& Emotion 24:810-828.

Lane AM, Devonport TJ, Friesen AP, Beedie CJ, Fullerton CL, and Stanley DM. 2015. How should I regulate my emotions if I want to run faster? European Journal of Sport Science 16:465.

Peer) reviewing PDF | (2019:04:37159:2:0:NEW 27 Jul 2019) 
585

586

587

588

589

590

591

592

593

594

595

596

597

598

599

600

601

602

603

604

605

606

607

608

609

610

611

612

613

614

615

616

617

618

619

620

621

622

623

624

625

626

627

628

629

630

631

632

633

634

635

Lang PJ. 1999. International Affective Picture System (IAPS) : Technical Manual and Affective Ratings. Center for Research in Psychophysiology University of Florida.

Lang PJ, Bradley MM, and Cuthbert BN. 1998. Emotion, motivation, and anxiety: brain mechanisms and psychophysiology. Biological Psychiatry 44:1248-1263.

Larson CL, Ruffalo D, Nietert JY, and Davidson RJ. 2005. Stability of emotion-modulated startle during short and long picture presentation. Psychophysiology 42:604-610.

Leuthold H, Sommer W, and Ulrich R. 2006. Preparing for action: inferences from CNV and LRP. Journal of Psychophysiology 18:77-88.

Loveless NE, and Sanford AJ. 1974. Slow potential correlates of preparatory set. Biological Psychology 1:303-314.

Lu B, Hui M, and Yuxia H. 2005. The Development of Native Chinese Affective Picture System-A pretest in 46 College Students. Chinese Mental Health Journal 19(11):719-722.

Lu Y, Luo Y, Lei Y, Jaquess KJ, Zhou C, and Li H. 2015. Decomposing valence intensity effects in disgusting and fearful stimuli: an event-related potential study. Social Neuroscience 11:618-626.

Luo W, Feng W, He W, Wang N-Y, and Luo Y-J. 2010. Three stages of facial expression processing: ERP study with rapid serial visual presentation. Neurolmage 49:1857-1867.

Müller MM, and Gundlach C. 2017. Competition for attentional resources between low spatial frequency content of emotional images and a foreground task in early visual cortex. Psychophysiology 54:429-443.

Müller MM, Tedersälejärvi W, and Hillyard SA. 1998. The time course of cortical facilitation during cued shifts of spatial attention. Nature Neuroscience 1:631-634.

Maxwell JS, and Davidson RJ. 2010. Emotion as motion: asymmetries in approach and avoidant actions. Psychological Science 18:1113-1119.

Melcher T, Obst K, Mann A, Paulus C, and Gruber O. 2012. Antagonistic modulatory influences of negative affect on cognitive control: Reduced and enhanced interference resolution capability after the induction of fear and sadness. Acta Psychologica 139:507-514.

Meng X, Liu W, Zhang L, Li X, Yao B, Ding X, Yuan JJ, and Yang J. 2016. EEG Oscillation Evidences of Enhanced Susceptibility to Emotional Stimuli during Adolescence. Frontiers in Psychology 7.

Michalak J, Troje NF, Fischer J, Vollmar P, Heidenreich T, and Schulte D. 2009. Embodiment of sadness and depression--gait patterns associated with dysphoric mood. Psychosomatic Medicine 71:580-587.

Pereira MG, Volchan E, de Souza GG, Oliveira L, Campagnoli RR, Pinheiro WM, and Pessoa L. 2006. Sustained and transient modulation of performance induced by emotional picture viewing. Emotion 6:622-634.

Pereira MG, Volchan E, Oliveira L, Machadopinheiro W, Rodrigues JA, Nepomuceno FV, and Pessoa L. 2004. Behavioral modulation by mutilation pictures in women. Brazilian Journal of Medical \& Biological Research 37:353-362.

Posner MI, and Petersen SE. 2012. The Attention System of the Human Brain. Annual Review of Neuroscience 13:25-42.

Pv L, Ben-David B, Lipski M, and Namasivayam A. 2014. The impact of threat and cognitive stress on speech motor control in people who stutter. Journal of Fluency Disorders 40:93-109.

Rathschlag M, and Memmert D. 2013. The influence of self-generated emotions on physical performance: an investigation of happiness, anger, anxiety, and sadness. J Sport Exerc Psychol 35:197-210.

Rathschlag M, and Memmert D. 2015. Self-Generated Emotions and Their Influence on Sprint Performance: An Investigation of Happiness and Anxiety. Journal of Applied Sport Psychology 27:186-199.

Rockstroh B, Elbert T, Birbaumer N, and Lutzenberger W. 1982. Slow brain potentials and

Peer) reviewing PDF | (2019:04:37159:2:0:NEW 27 Jul 2019) 
636

637

638

639

640

641

642

643

644

645

646

647

648

649

650

651

652

653

654

655

656

657

658

659

660

661

662

663

664

665

666

667

668

669

670

671

672

673

674

675

676

677

678

679

680

behavior.

Rohrbaugh JW, Syndulko K, ., and Lindsley DB. 1976. Brain wave components of the contingent negative variation in humans. Science 191:1055-1057.

Rotteveel M, and Phaf $\mathrm{RH}$. 2004. Automatic affective evaluation does not automatically predispose for arm flexion and extension. Emotion 4:156-172.

Shaw EP, Rietschel JC, Hendershot BD, Pruziner AL, Miller MW, Hatfield BD, and Gentili RJ. 2018. Measurement of attentional reserve and mental effort for cognitive workload assessment under various task demands during dual-task walking. Biological Psychology 134.

Simoes Matos Saraiva A. 2017. Motion and Emotion: How emotional stimuli influence the motor system. UCL (University College London).

Stoodley CJ, and Schmahmann JD. 2010. Evidence for topographic organization in the cerebellum of motor control versus cognitive and affective processing. Cortex 46:831844.

Sun J, Sun B, Wang B, and Gong H. 2012. The processing bias for threatening cues revealed by event-related potential and event-related oscillation analyses. Neuroscience 203:9198.

Tallon-Baudry C, Bertrand O, Delpuech C, and Pernier J. 1996. Stimulus specificity of phaselocked and non-phase-locked $40 \mathrm{~Hz}$ visual responses in human. Journal of Neuroscience 16:4240-4249.

Taylor MJ. 2002. Non-spatial attentional effects on P1. Clinical neurophysiology 113:1903-1908.

Tecce JJ. 1972. Contingent negative variation (CNV) and psychological processes in man. Psychological Bulletin 77:73-108.

Van dSS, Imants P, and Ridderinkhof KR. 2011. Positive affect increases cognitive control in the antisaccade task. Brain \& Cognition 75:177-181.

Vanlessen N, Raedt RD, Mueller SC, Rossi V, and Pourtois G. 2015. Happy and less inhibited? Effects of positive mood on inhibitory control during an antisaccade task revealed using topographic evoked potential mapping. Biological Psychology 110:190-200.

Walter WG. 1964. The contingent negative variation: An electrocortical sign of significant association in the human brain.

Wong TKW, Fung PCW, Mcalonan GM, and Chua SE. 2009. Spatiotemporal dipole source localization of face processing ERPs in adolescents: a preliminary study. Behavioral \& Brain Functions 5:16.

Yuan J, Zhang Q, Chen A, Li H, Wang Q, Zhuang Z, and Jia S. 2007. Are we sensitive to valence differences in emotionally negative stimuli? Electrophysiological evidence from an ERP study. Neuropsychologia 45:2764-2771.

Zhang D, Liu Y, Wang X, Chen Y, and Luo Y. 2014. The duration of disgusted and fearful faces is judged longer and shorter than that of neutral faces: The attention-related time distortions as revealed by behavioral and electrophysiological measurements. Frontiers in Behavioral Neuroscience 8:293.

Zhang G, Tian L, Chen H, Li P, Ristaniemi T, Wang H, Li H, Chen H, and Cong F. 2017. Effect of parametric variation of center frequency and bandwidth of morlet wavelet transform on time-frequency analysis of event-related potentials. Chinese Intelligent Systems Conference: Springer. p 693-702. 
Figure 1

The cued-action task.

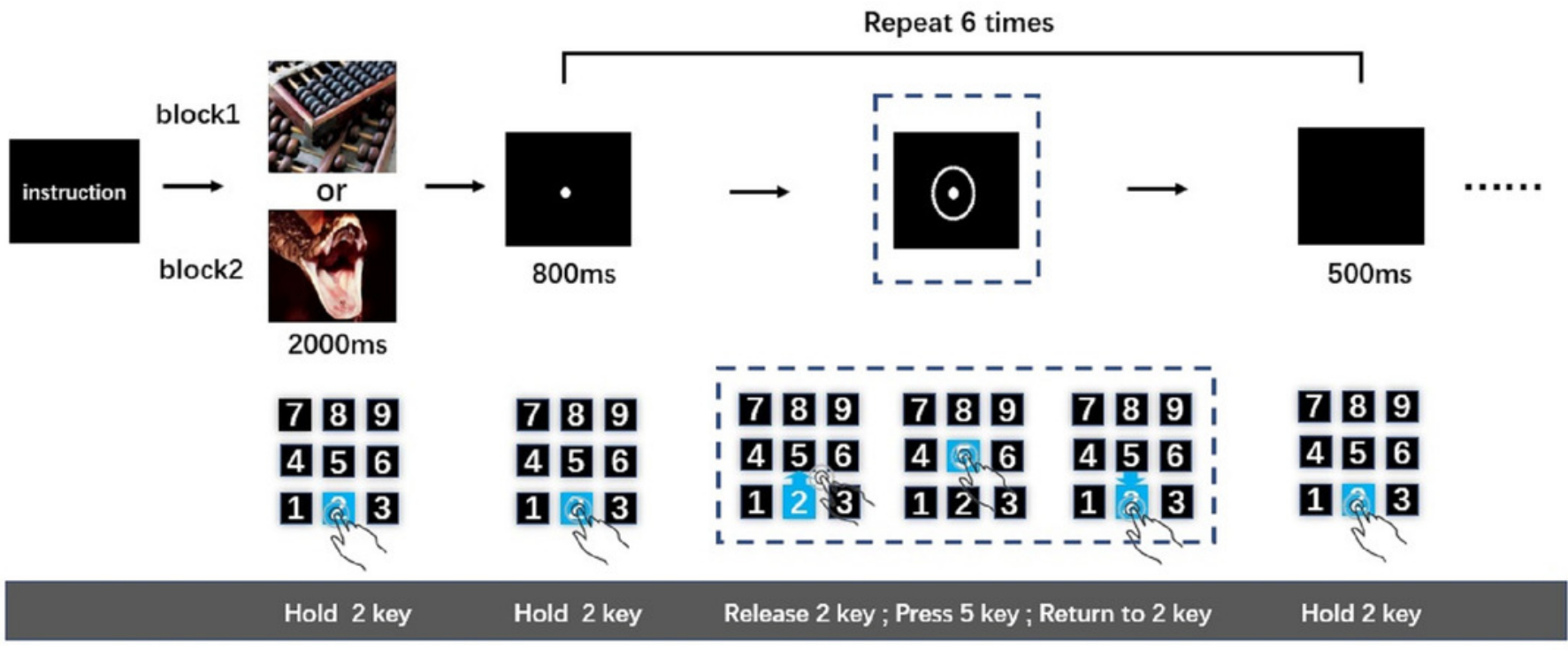




\section{Figure 2}

Action time $\square 2 \times 2$ ANOVA $\square$ and SAM scores under different experimental conditions.

(A) A histogram of SAM score under neutral and negative conditions. (B) The scatter plots with box plots of SAM score. (C) A histogram of action time under different experimental conditions. (D) The scatter plots with box plots of action time.
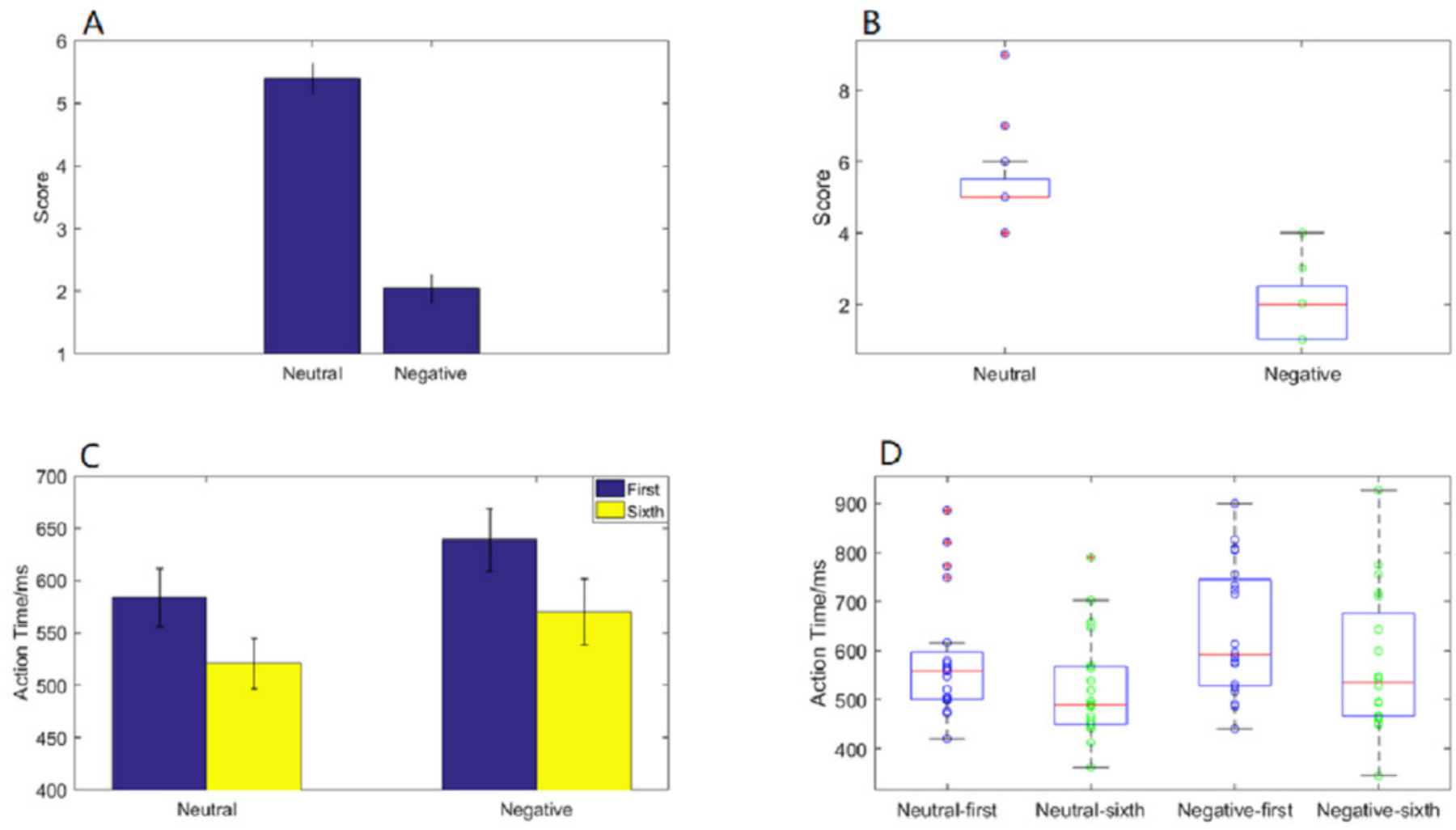
Figure 3

Action time under different experimental conditions(six levels in the action sequence factor).

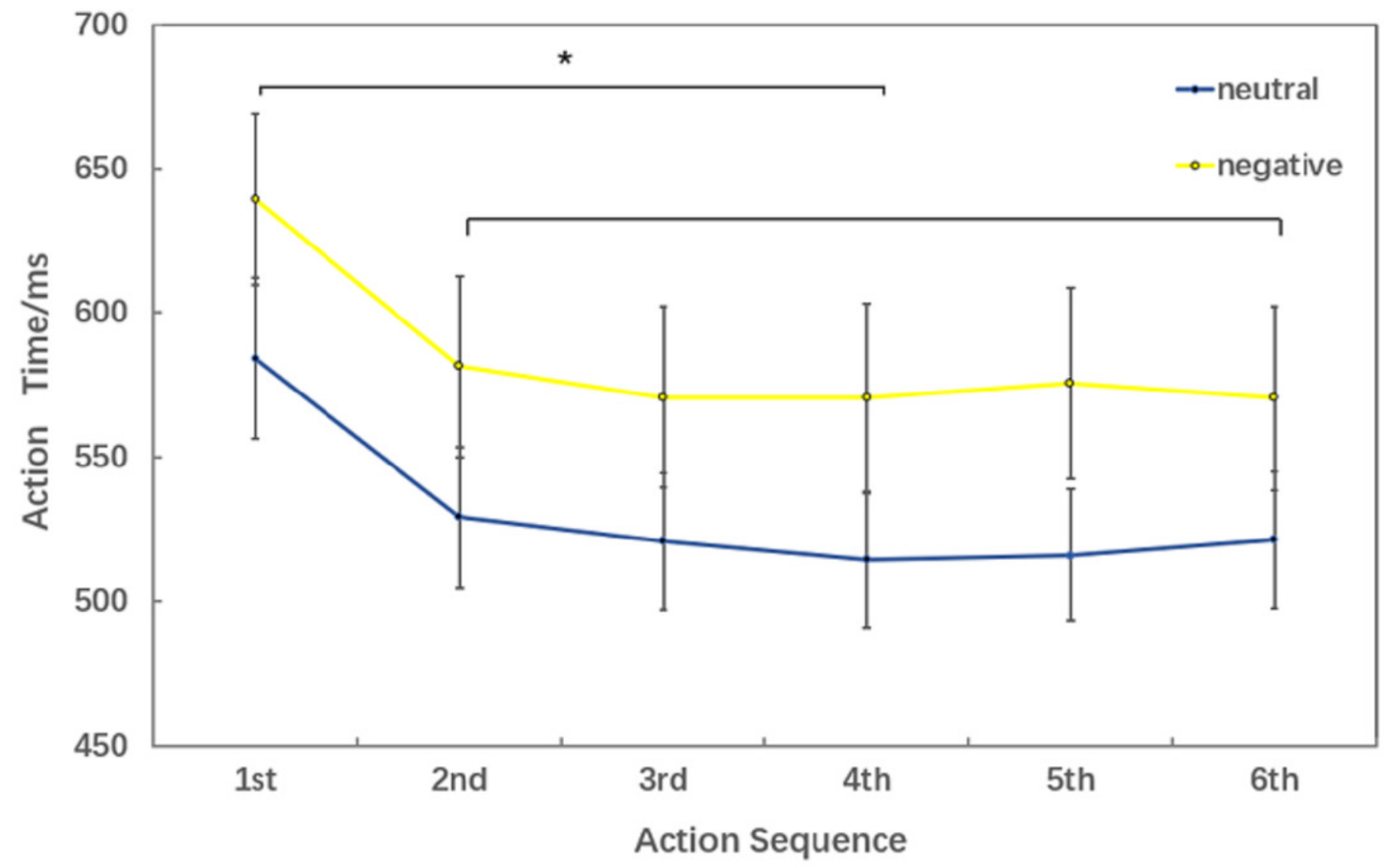


Figure 4

P1 component elicited under neutral and negative conditions at electrode sites P03, P04, PO7, PO8, POz, $\mathrm{O} 1,02, \mathrm{Oz}$ and Brain topography of P1.

(A)The P1 component elicited by neutral and negative conditions. (B) Brain topography of P1 under negative condition. (C) Brain topography of P1 under neutral condition.

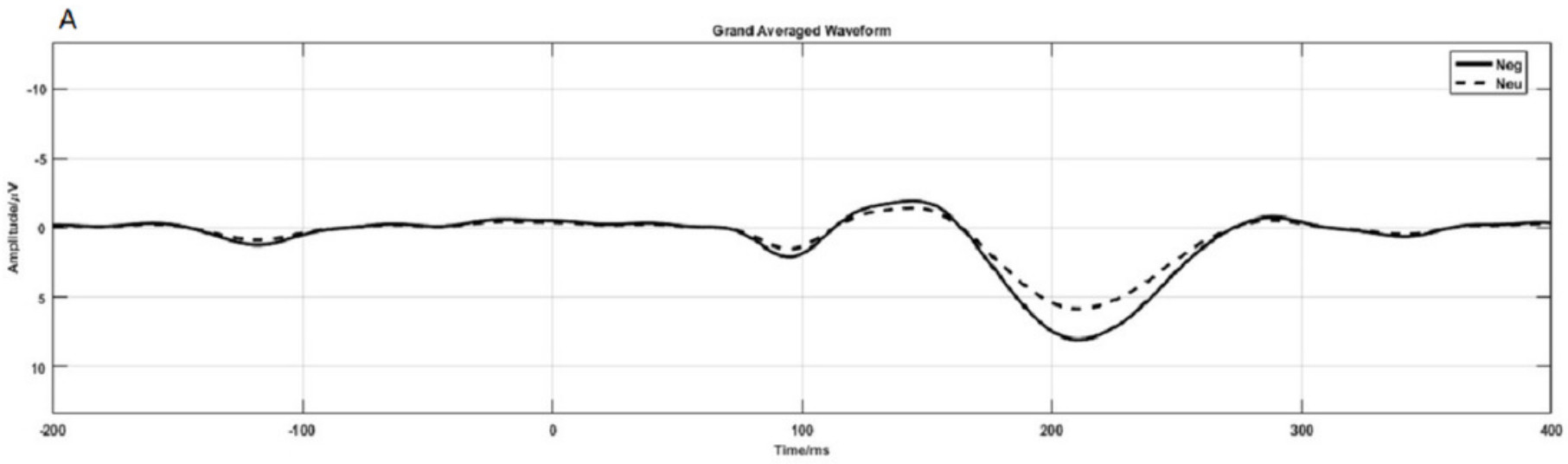

B
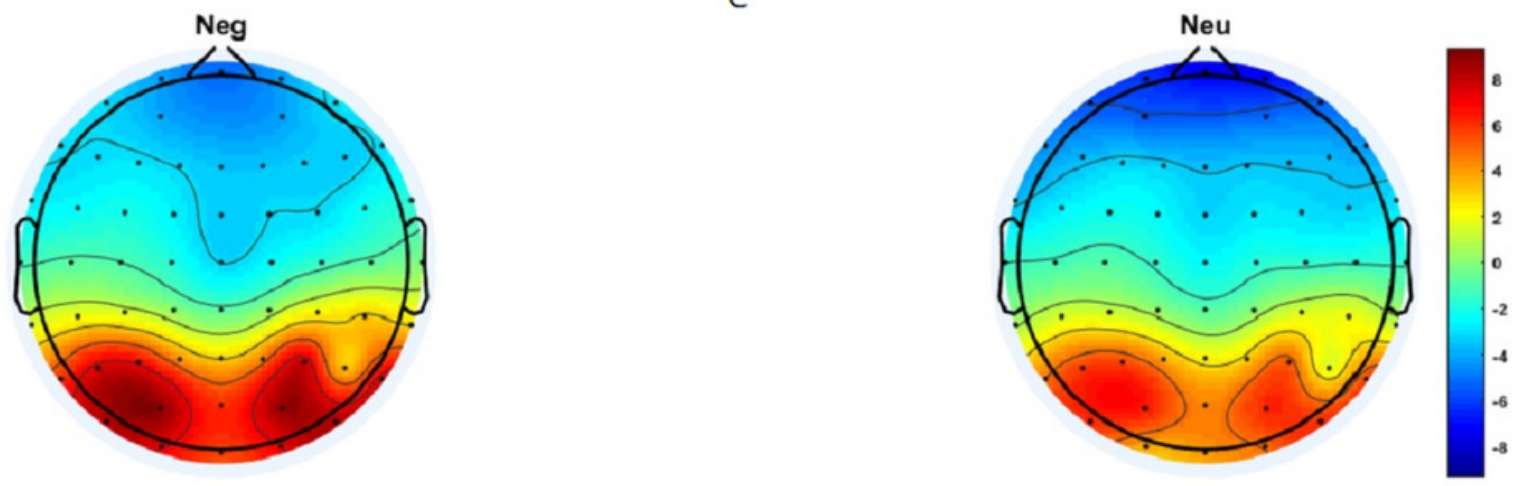


\section{Figure 5}

Theta oscillationelicited under neutral and negative conditions at electrode sites PO3, PO4, PO7, PO8 , POz, $\mathrm{O} 1, \mathrm{O} 2, \mathrm{Oz}$ andBrain topography of Theta oscillation.

(A) Theta oscillation elicited by negative condition. (B) Theta oscillation elicited by neutral condition. (C) Brain topography of theta oscillation under negative condition. (D) Brain topography of theta oscillation under neutral condition.

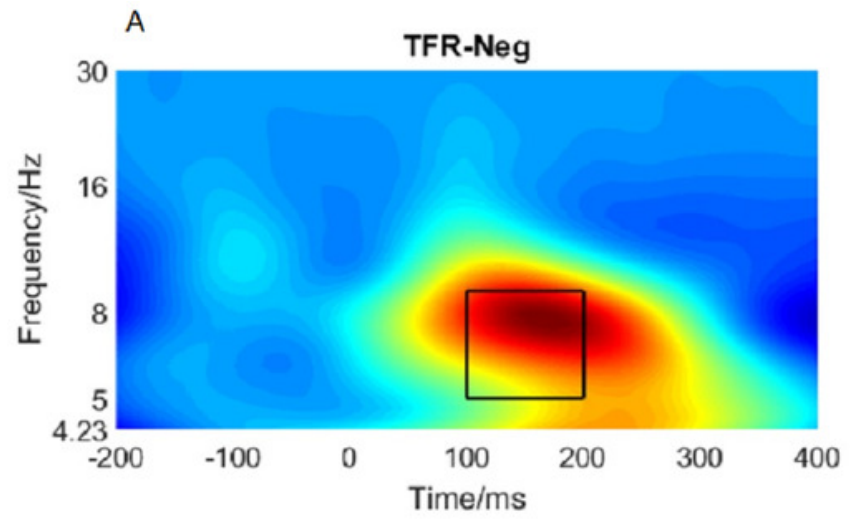

C

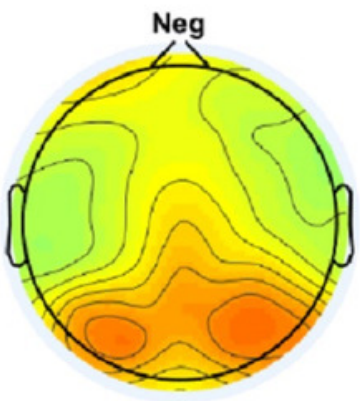

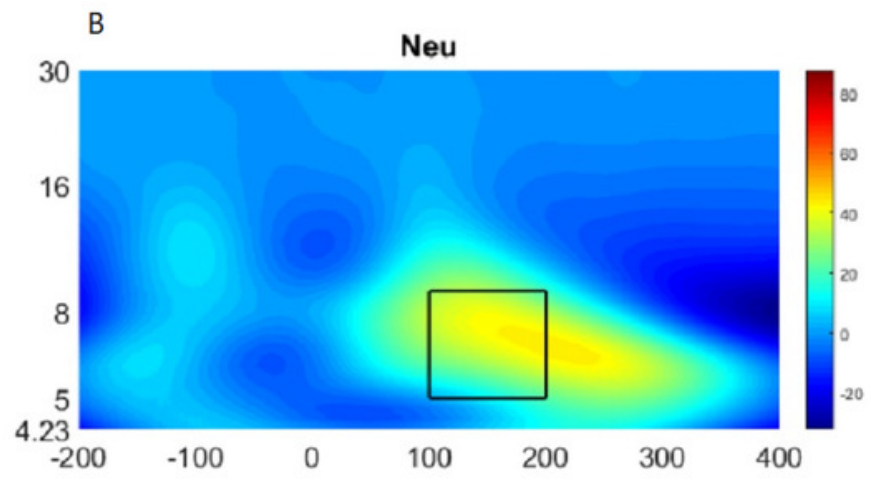

D

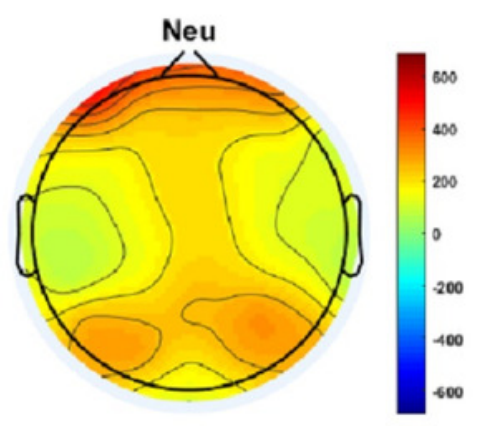




\section{Figure 6}

The early CNV and late CNV elicited under different conditions at electrode sites Fz, FCz and Brain topography of early CNV and late CNV, respectively.

(A) The early C NV under different conditions at Fz. (B) The early C NV under different conditions at FCz. (C) Brain topography of early CNV at negative emotion and 1st action task. (D) Brain topography of early CNV at negative emotion and 6th action task. (E) Brain topography of early CNV at neutral emotion and 1st action task. (F) Brain topography of early CNV at neutral emotion and 6th action task. (G) Brain topography of late CNV at negative emotion and 1st action task. (H) Brain topography of late CNV at negative emotion and 6th action task. (I) Brain topography of late CNV at neutral emotion and 1st action task. (J) Brain topography of late CNV at neutral emotion and 6th action task.
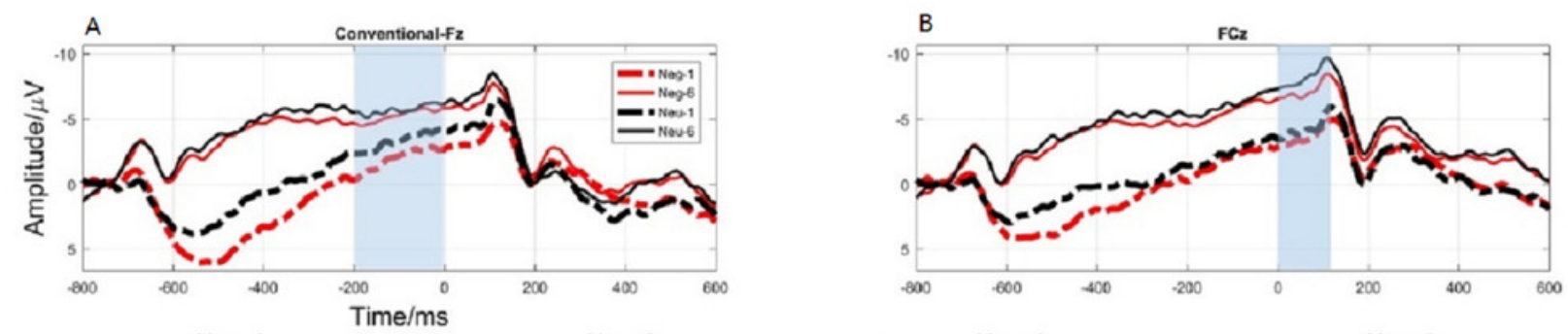

C

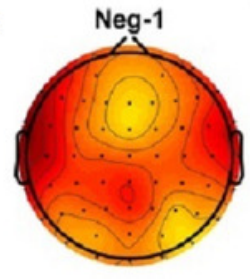

G

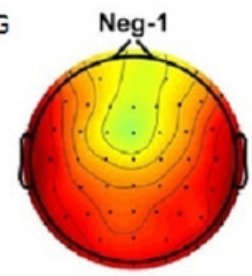

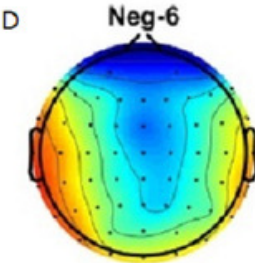

H

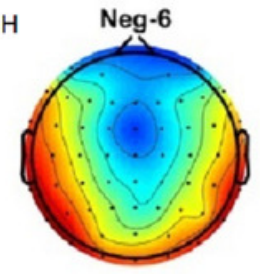

E
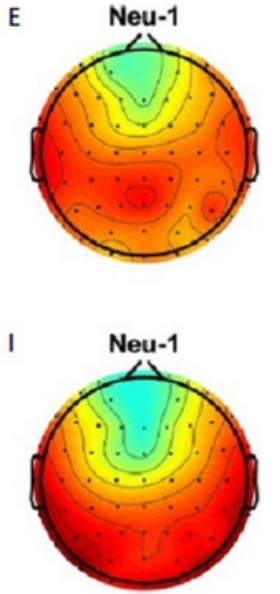

$\mathrm{F}$
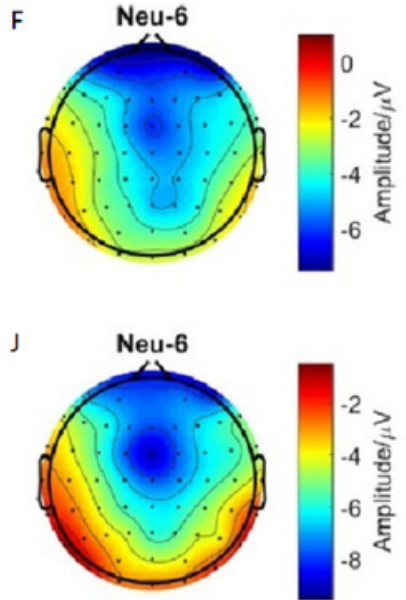


\section{Table $\mathbf{1}$ (on next page)}

The SAM Ratings of emotional pictures 
1 The SAM Ratings of emotional pictures

\begin{tabular}{lll}
\hline & Valence & Arousal \\
Emotion type & $M(\mathrm{SD})$ & $M(\mathrm{SD})$ \\
\hline Negative & $2.63(0.82)$ & $5.88(1.07)$ \\
Neutral & $5.03(0.13)$ & $3.70(1.20)$ \\
\hline
\end{tabular}

